\title{
APPROXIMATE EQUIVALENCE OF REPRESENTATIONS OF AF ALGEBRAS INTO SEMIFINITE VON NEUMANN ALGEBRAS
}

\author{
Junsheng FAng, Rui Shi And Shilin WEN
}

Abstract. In this paper, we extend the "compact operator" part of Voiculescu's theorem on approximate equivalence of unital $*$-homomorphisms of an AF algebra when the range is in a countably decomposable, semifinite, properly infinite von Neumann factor. We also extend a result of Hadwin for approximate summands of representations into a finite von Neumann factor.

Mathematics subject classification (2010): 47C15.

Keywords and phrases: Weyl-von Neumann theorem, Voiculescu's theorem, AF algebras, countably decomposable semifinite von Neumann algebras.

\section{REFERENCES}

[1] William Arveson, Notes on extensions of $C^{*}$-algebras, Duke Math. J. 44 (1977), no. 2, 329-355.

[2] DAVID BERG, An extension of the Weyl-von Neumann theorem to normal operators, Trans. Amer. Math. Soc. 160 (1971), 365-371.

[3] Alin Ciuperca, Thierry Giordano, Ping Wong Ng and Zhuang Niu, Amenability and uniqueness, Adv. Math. 240 (2013), 325-345.

[4] Kenneth Davidson, $C^{*}$-algebras by example, Fields Institute Monographs, 6. American Mathematical Society, Providence, RI, 1996.

[5] Kenneth Davidson, Normal operators are diagonal plus Hilbert-Schmidt, J. Operator Theory 20 (1988), no. 2, 241-249.

[6] Huiru Ding And Don Hadwin, Approximate equivalence in von Neumann algebras, Sci. China Ser. A 48 (2005), no. 2, 239-247.

[7] Donald Hadwin, Nonseparable approximate equivalence, Trans. Amer. Math. Soc. 266 (1981), no. $1,203-231$.

[8] Donald Hadwin AND RUI SHI, A note on representations of commutative $C^{*}$-algebras in semifinite von Neumann algebras, Oper. and Matrices , 12 (2018), no. 4, 1129-1144.

[9] Paul Halmos, Ten problems in Hilbert space, Bull. Amer. Math. Soc. 76 (1970), 887-933.

[10] Richard Kadison And John Ringrose, Fundamentals of the theory of operator algebras. Vol. I. Elementary theory, Reprint of the 1983 original, Graduate Studies in Mathematics, 15. American Mathematical Society, Providence, RI, 1997.

[11] Richard Kadison And John Ringrose, Fundamentals of the theory of operator algebras. Vol. II. Advanced theory, Corrected reprint of the 1986 original, Graduate Studies in Mathematics, 16. American Mathematical Society, Providence, RI, 1997.

[12] Victor Kaftal, On the theory of compact operators in von Neumann algebras. II, Pacific J. Math. 79 (1978), no. 1, 129-137.

[13] Qinui Li, Junhao Shen, Rui Shi, A generalization of the Voiculescu theorem for normal operators in semifinite von Neumann algebras, arXiv:1706.09522 [math.OA].

[14] Qinui Li, Junhao Shen, Rui Shi, Liguang Wang, Perturbations of self-adjoint operators in semifinite von Neumann algebras: Kato-Rosenblum theorem, J. Funct. Anal. 275 (2018), no. 2, 259287.

[15] John von Neumann, Charakterisierung des Spektrums eines Integraloperators, Actualits Sci. Indust. 229, Hermann, Paris, 1935. 
[16] Mikael Rørdam, Flemming Larsen and Niels Jakob Laustsen, An introduction to $K$ theory for $\mathrm{C}^{*}$-algebras, London Mathematical Society Student Texts, 49. Cambridge University Press, Cambridge, 2000.

[17] MASAmichi TAKeSAKI, Theory of operator algebras. I. Reprint of the first (1979) edition, Encyclopaedia of Mathematical Sciences, 124. Operator Algebras and Non-commutative Geometry, 5. Springer-Verlag, Berlin, 2002.

[18] Dan Voiculescu, A non-commutative Weyl-von Neumann theorem, Rev. Roumaine Math. Pures Appl. 21 (1976), no. 1, 97-113.

[19] DAN VOICULESCU, Some results on norm-ideal perturbations of Hilbert space operators, J. Operator Theory 2 (1979), no. 1, 3-37.

[20] Hermann WeYl, Über beschränkte quadratische formen, deren differenz vollstetig ist, Rend. Circ. Mat. Palermo 27 (1) (1909), 373-392.

[21] LÁszLó Zsidó, The Weyl-von Neumann theorem in semifinite factors, J. Funct. Anal. 18 (1975), 60-72. 